
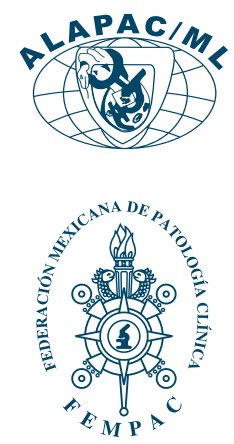

\section{Bioética en tiempos de la pandemia}

\author{
Bioethics in times of pandemic
}

García-Solís Eduardo*

Palabras clave: Bioética, pandemia, SARS-CoV-2.

Keywords: Bioethics, pandemic, SARS-COV-2.

\footnotetext{
* Médico Cirujano con Especialidad en Patología Clínica. Director Ejecutivo de la Comisión de Bioética del Estado de Campeche. México.
}

Correspondencia: Eduardo García-Solís Calle $49 \mathrm{~s} / \mathrm{n}$, Altos del Hospital «Dr. Manuel Campos», Barrio de Guadalupe, 24010,

San Francisco de Campeche, Campeche, México. Teléfono: 98 1829-9647

E-mail: egarciasolis@ hotmail.com

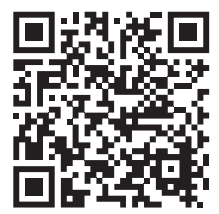

\section{RESUMEN}

La pandemia actual causada por el coronavirus (SARSCoV-2), identificada en Wuhan, China, en diciembre de 2019, se ha propagado en el mundo. Primero por Europa, posteriormente en Estados Unidos y finalmente en América Latina. Ha ocasionado el colapso de sistemas de salud, principalmente por falta de ventiladores, equipo de protección personal, camas en unidades de cuidados intensivos y personal adiestrado en medicina crítica. La enfermedad llegó a México el 23 de febrero de 2020 y se han aplicado medidas de prevención como la sana distancia, el lavado frecuente de manos, permanecer en casa para aplanar la curva de propagación con el objetivo de salvar vidas. Con la participación de la bioética en la epidemia se intenta hacer frente a las tensiones y conflictos que derivan del choque de valores entre culturas, intereses individuales y colectivos. Se debe poner de relieve la protección de grupos en condiciones de vulnerabilidad. Se deben transformar los sistemas de salud e impulsar iniciativas que contribuyan a superar la exclusión, la inequidad y las barreras al acceso y uso oportuno de servicios de salud integrales. Esta es una tarea que tienen todos los que participan en el sector salud.

Con afecto al Excelente médico, mejor ser humano, con la paciencia de Job.

\section{INTRODUCCIÓN}

$\Pi \mathrm{n}$ estos tiempos de pandemia, es la contingencia causada por el coronavirus (SARS-CoV-2) la que nos ha hecho vivir situaciones que probablemente han afectado nuestro día a día y nuestra forma de vivir. Esta enfermedad fue identificada primero en la ciudad de Wuhan, China, en diciembre de 2019, la cual evolucionó rápidamente a Dr. Hugo López-Gatell Ramírez org/10.35366/99464

\section{ABSTRACT}

The current pandemic caused by the coronavirus (SARSCoV-2), first identified in the Chinese city of Wuhan on December 2019, has propagated around the world. First Europe, posteriorly USA, and finally Latin America. It caused the collapse of health systems, mainly because the lack of ventilators, personal protection equipment, beds in ICUs and personal trained in critical care. The disease arrived in Mexico on February 23, 2020 and several measures have been implemented such as "healthy distance», frequent hand wash, and stay at home, this in order to flatten the curve with the purpose of saving lives. With the involvement of bioethics in the epidemic, we try to face the tensions and conflicts that arise from the clash between cultures, individual and collective interests. The protection of vulnerable groups must be highlighted. Health systems must be transformed and give impulse to initiatives that help ameliorate the exclusion, inequity and barriers to get the integral health interventions in a timely manner. This is a task everyone in the health system have.

Citar como: García-Solís E. Bioética en tiempos de la pandemia. Rev Mex Patol Clin Med Lab. 2020; 67 (4): 183-184. https://dx.doi. 
La bioética tiene un papel preponderante: reflexiona, delibera y hace planteamientos normativos y de políticas públicas para regular y resolver conflictos en la vida social, especialmente en las ciencias de la vida, así como en la práctica y en la investigación médica que afecten la vida en el planeta, tanto en la actualidad como en futuras generaciones. ${ }^{2} \mathrm{Al}$ considerar a la bioética como un marco de referencia para saber cómo actuar ante una pandemia, resulta un diléma bioético el uso de escalas para tomar una decisión que influirá en la supervivencia del paciente, nada más difícil. ${ }^{3}$ En el ámbito global, la bioética se ha posicionado como un quehacer inter- $y$ multidisciplinario que promueve un enfoque integral en el abordaje de la salud, con el fin de hacer frente a las tensiones y conflictos que deriven del choque de valores entre culturas, considerando la ponderación entre intereses individuales y colectivos, poniendo de relieve la protección de grupos en condiciones de vulnerabilidad. ${ }^{4}$

Van Rensselaer Potter hace 50 años escribía el libro «Bridge to future», ${ }^{5}$ dando a conocer a la bioética, considerando a ésta para referirse a la disciplina que combina el conocimiento biológico y los valores humanos: la ciencia de la supervivencia y puente hacia el futuro. Potter pensaba que la bioética era una nueva cultura, el encuentro necesario entre la vida y los valores; entre las ciencias y las humanidades. Considerar el significado original de la bioética, entendiéndola como la reflexión moral acerca de las cuestiones biomédicas, la crisis ecológica y la distribución de recursos. Los bioeticistas están obligados a considerar no sólo las decisiones cotidianas, sino también las consecuencias a largo plazo de las acciones que recomendaron o que dejaron de considerar. ${ }^{6}$ Por lo que la bioética es responsable del análisis, discusión y apoyo en la toma de decisiones respecto a los problemas bioéticos que se presentan en la práctica clínica, la atención médica, la docencia y la investigación en el área de la salud. En la actualidad, la presencia de la pandemia por el SARS-CoV-2 ha causado tantos estragos en la población en el mes de diciembre de 2020, con más de 100,000 fallecidos. Cabe señalar que tal pandemia no se esperaba y que el mundo no estaba preparado.

En el México reciente se presentaron las epidemias de la gripe española, el cólera, la influenza, pero nada comparado con la pandemia actual. Por lo que se deben transformar los sistemas de salud e impulsar iniciativas que contribuyan a superar la exclusión, la inequidad y las barreras al acceso y uso oportuno de servicios de salud integrales, esta es una tarea que tienen todos los que participan en el sector salud. Hace ya 40 años de la declaración de Alma Ata y no se ha avanzado en el combate de la inequidad en la atención a la salud, se han acentuado diversos mecanismos como la segmentación y fragmentación de los sistemas de salud, lo que ha ocasionado que las personas reciban atención médica dependiendo de su lugar laboral o de su capacidad de pago de bolsillo, provocando que quienes tienen más recursos puedan acceder a mejores servicios. Además, hay una multiplicidad de infraestructura médica, lo que ha provocado la centralización del tratamiento de los padecimientos, en lugar de atender de manera integral la salud del individuo.

Por último, en la atención médica diaria se da la relación médico-paciente, la mayor parte de las veces satisfactoria, aunque en ocasiones no es así, rompiéndose esa relación ya sea por negligencia, impericia o imprudencia. Y muchas veces por falta de humanismo, de comunicación. Es en estos tiempos de pandemia en los que el ser humano necesita del ser humano, en los que el equipo de salud debe contar con las medidas de seguridad máximas y el no contar con ello significa dejar en indefensión a todos. Las autoridades gubernamentales, así como la población, deben ser conscientes, corresponsables y acatar las medidas sanitarias. Es tarea de todos, seguramente no es un camino fácil, y habrá que encontrar los caminos.

En la epidemia causada por el SARS-CoV-2 la bioética tiene un papel preponderante. Seamos los buenos antepasados de nuestros descendientes.

\section{REFERENCIAS}

1. Zhu N, Zhang D, Wang W, Li X, Yang B, Song J et al. A novel coronavirus from patients with pneumonia in China, 2019. N Engl J Med. 2020; 382 (8): 727-733.

2. Guía Nacional para la Integración y el Funcionamiento de los Comités Hospitalarios de Bioética. 2015. Disponible en: http://www.conbioetica-mexico.salud.gob.mx/descargas/pdf/ registrocomites/Guia_CHB_Final_Paginada_con_forros.pdf

3. Consejo de Salubridad General. Guía bioética para asignación de recursos limitados de medicina crítica en situación de emergencia. Ciudad de México: CSG; 2020. Disponible en: http:// www.csg.gob.mx/descargas/pdf/index/informacion_relevante/ GuiaBioeticaTriaje_30_Abril_2020_7pm.pdf

4. Comisión Nacional de Bioética. Pronunciamiento ante la pandemia del COVID-19. Disponible en: www.gob.mx/salud/conbioetica

5. Potter VR. Bioethics: the science of survival. Perspectives in Biology and Medicine. 1970;(14):127-153.

6. Amor-Pan JR. Bioética: un puente hacia el futuro. Disponible: www. fpablovi.org/index.php/articulos/976-bioetica-unpuente-haciaelfuturo 